Revista Universo Contábil, ISSN 1809-3337

FURB, v. 5, n. 2, p. 142-157, abr./jun., 2009

doi:10.4270/ruc. 2009218

Disponível em www.furb.br/universocontabil

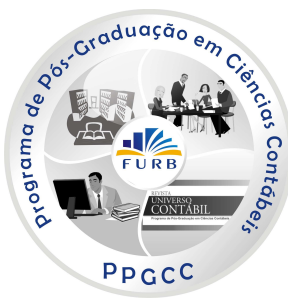

\title{
LOS NUEVOS DESAFÍOS DE LA CONSULTORÍA ECONÓMICA: CONTRASTACIÓN EMPÍRICA*
}

\section{THE NEW CHALLENGES OF ECONOMIC CONSULTANCY: AN EMPIRIC INVESTIGATION}

Maria Mercedes Carro Arana

Doctora y Profesora Titular de la

Facultad de Ciencias Económicas y Empresariales de la Universidad de Cantabria

Endereço: Av. Los Castros s/n CEP: 39005 - Santander - España E-mail: mercedes.carro@unican.es Teléfono: +34942 201655

\section{Gemma Hernando Moliner}

Doctora y Profesora Titular de la Facultad de Ciencias Económicas y Empresariales de la Universidad de Cantabria Endereço: Av. Los Castros s/n CEP: 39005 - Santander - España E-mail: gemma.hernando@unican.es Teléfono: +34942 201655

\section{RESUMEN}

El objetivo de este trabajo ha consistido en analizar los nuevos retos que plantea el campo de la consultoría económica, ante la evolución de su demanda tanto en España como en la Unión Europea. Se estudia la adecuación que debe darse en las consultorías para satisfacer las necesidades externalizadas por las empresas en relación a sus funciones administrativas y de gestión. Se realiza una revisión crítica de las necesidades de asesoramiento empresarial y

\footnotetext{
Artigo recebido em 24.01.2007. Revisado por pares em 22.03.2007. Reformulado em 11.11.2007. Recomendado em 15.12.2007 por Ilse Maria Beuren (Editora). Publicado em 30.06.2009. Organização responsável pelo periódico: FURB.
} 
un doble estudio empírico, basado en dos encuestas: una captando la percepción de los empresarios y la otra, de los profesionales. El estudio evidencia la dependencia que tienen las empresas de las consultorías. La necesidad de mejorar la formación de los profesionales y su estructura organizativa, así como de cuidar la ética, la calidad y responsabilidad asumida. Se muestran también las ventajas de crear despachos multidisciplinares que puedan ofrecer servicios integrales, destacándose la conveniencia de ofrecer servicios más especializados, resaltándose los de tecnología de la información e integración de sistemas.

Palabras claves: Desafíos. Consultoría Económica. Contrastación Emprírica.

\section{RESUMO}

O objetivo do trabalho consiste em analisar os novos desafios que exige o campo da consultoria econômica, diante da evolução da sua demanda tanto na Espanha como na União Européia. Estuda-se a adequação que se deve dar às consultorias para satisfazer as necessidades expressadas pelas empresas com relação as suas funções administrativas e de gestão. Realiza-se uma revisão crítica das necessidades de assessoramento empresarial e um duplo estudo empírico, com base em dois questionários: um captando a percepção dos empresários e outro a dos profissionais de consultoria. O estudo evidencia a dependência que as empresas têm da consultoria. Por outro lado, mostra a necessidade de melhorar a formação dos profissionais e a sua estrutura organizacional, assim como de cuidar da ética, da qualidade e responsabilidade assumida. Mostram-se também as vantagens de criar escritórios multidisciplinares que possam oferecer serviços integrais, destacando-se a conveniência de oferecer serviços mais especializados, ressaltando-se os de tecnologia da informação e integração de sistemas.

Palavras-chave: Desafios. Consultoria Econômica. Contrastação Empírica.

\section{ABSTRACT}

The objective of this paper is to analyse the new challenges required by economic consultancy area, due to the evolution of its demand both in Spain and in the European Union. The adequation that should be done to the consultancies to satisfy the necessities of the companies for the administrative and management functions is studied. This paper carries out a critical revision of the necessities of managerial advice and a double empiric investigation, based on two surveys: one capturing the perception of the managers and the other one, of the business professionals. The study evidences the dependence that companies have on economic consultancy entities. The necessity to improve the professionals qualification and their organizational structure, as well as taking care of the ethics, the quality and assumed responsibility. Also, the advantages of creating consultancy that can offer integral services (business, financial, accounting, tax, labor...) are presented, outlining the convenience of offering specialized services, especially those of information technology and systems integration.

Keywords: Challenges. Economic consultancy. Empirical investigation. 


\section{INTRODUCCIÓN}

Los datos estadísticos oficiales muestran que la evolución de la demanda de los servicios de consultoría económica se ha potenciado en los últimos años, tanto en España como en la Unión Europea. Ello se debe principalmente al crecimiento económico y a la complejidad y responsabilidad que revisten los negocios de hoy en día.

El papel que en la actualidad tienen los consultores económicos y los nuevos desafíos con que deben enfrentarse se puede analizar a la vista de las necesidades que están planteando las empresas así como ante la demanda de colaboración de profesionales expertos. Ello supone, de un lado estudiar dichas necesidades y de otra parte, analizar los atributos o competencias básicas que en la época actual son exigibles a estos profesionales, así como la utilidad y calidad de los servicios ofrecidos.

En lo que se refiere a los atributos personales, en nuestra opinión, pueden resumirse en dos: Por una parte, deben poseer el conocimiento necesario (fruto de la cualificación y la experiencia) que permita "saber qué hacer" ante la necesidad específica que le plantea la empresa; y, por otra parte, deben tener la independencia y la objetividad necesarias para responder al "cómo hacerlo" en una organización específica. A ello, nos parece preciso añadir, una cualidad igualmente exigible al consultor económico de nuestros días, como es, un comportamiento ético.

En la sociedad actual la calidad se ha convertido en el objetivo prioritario de todas las actividades empresariales y profesionales. Sin embargo la calidad en las entidades de consultoría no podemos entenderla como un concepto técnico, sino más bien como una filosofía de gestión que abarca a todas las actividades del proceso completo del servicio que prestan los despachos profesionales. El cliente es cada vez más exigente. Ya no se conforma con la prestación de unos servicios sino que exige lo mejor. Además sabe que el mercado de las asesorías, consultorías o bufetes es muy amplio y le sobra donde escoger. Por ello, los despachos de consultoría deben responder a los retos que plantea la sociedad actual y sus clientes, adaptándose a sus necesidades y contribuyendo a su evolución, buscando ser capaces de prestar unos servicios eficaces, responsables y rentables.

El objetivo de este estudio ha consistido en analizar los nuevos retos que se deben llevar a cabo en el sector de consultoría económica, al objeto de satisfacer las necesidades de externalización de funciones administrativas y de gestión que están demandando las empresas en la época actual. Para ello se ha realizado una revisión bibliográfica y estadística de los diferentes aspectos que contribuyen a explicar la realidad actual tanto en lo que se refiere a las necesidades de asesoramiento de las empresas como a la evolución del sector de consultoría económica. Posteriormente, se ha realizado un doble estudio empírico, basado en dos encuestas de opinión. Una ha permitido captar de forma directa la percepción de los empresarios y sus necesidades y la otra la percepción desde el punto de vista de los profesionales de la consultoría que atienden aquellas necesidades. Finalmente todo ello ha permitido contrastar y obtener algunos resultados que se muestran al final de este trabajo.

\section{EL SECTOR DE CONSULTORÍA ECONÓMICA: RASGOS CARACTERÍSTICOS}

Entendemos por consultoría económica la actividad desarrollada por profesionales, independientes a las entidades económicas-clientes, que cuentan con los medios y cualificación necesarios para llevar a cabo y/o colaborar en la ejecución eficiente de tareas de administración y de gestión de empresas y entidades, ya sean privadas o públicas. En 
consecuencia, la misión del asesor económico consiste en ayudar tanto en sus funciones de administración como en las de gestión de manera eficiente.

De ahí que, el campo de actuación del asesor económico abarque: Desde las actividades más clásicas de consultoría relacionadas con los ámbitos contable, financiero y fiscal, vinculadas a necesidades sistemáticas y principalmente relacionadas con el cumplimiento de normas; a las actividades relacionadas con los ámbitos de gestión y dirección, vinculadas a necesidades tanto específicas como sistemáticas y relacionadas con las distintas áreas de la empresa a gestionar.

Las diferentes líneas de servicios ofrecidos desde estas organizaciones han motivado la utilización de distintas denominaciones a las que nos referiremos a lo largo de este trabajo como son: consultorías, asesorías, gestorías y despachos profesionales entre otros, que ofrecen sus servicios tanto a empresas privadas, como a particulares y a entidades públicas.

Sin duda, el desarrollo de la consultoría económica está estrechamente relacionado con el desarrollo del orden empresarial en el que sirve. Así, hoy en día, el entorno de los despachos de consultoría económica, está marcado por diferentes hechos significativos como los siguientes:

a) En primer lugar, el sector se caracteriza por la multitud de normas que regulan la actividad empresarial en muchos casos impuestos por la normativa internacional que exige mayor capacidad de adaptación así como mayor formación y actualización permanentes en los profesionales. Las numerosas y complejas normas que se publican por año en cada materia contable, fiscal, laboral o mercantil hace prácticamente imposible aglutinar el conocimiento de todas ellas en un solo profesional. Este hecho esta dando lugar a la especialización de los profesionales así como a la creación de despachos multidisciplinares, desde los cuales pueda ofrecerse un servicio integral a la empresa como parece que en la actualidad se esta demandando.

b) En segundo lugar algunos Estados Miembros de la Unión Europea han reconocido la distinta realidad económica de la pequeña y la gran empresa y han creado profesiones distintas; una profesión que fundamentalmente esta involucrada en el asesoramiento de las microempresas, pequeñas y medianas y otra profesión que se concentra en grandes entidades y grupos empresariales, requiriéndose según los distintos países titulaciones y niveles universitarios diferentes. En todo caso, respetuosos con los códigos deontológico, con la calidad y con la formación.

c) En tercer lugar, el sector de consultoría, en común con el resto de las empresas, se ve afectado por la modificación de las formas de competir en sus mercados. El fenómeno de la globalización económica unido a la incorporación generalizada de las nuevas tecnologías de la información y comunicaciones ha intensificado el grado de competencia en unos mercados que ya lo venían experimentando ante las mayores exigencias de los clientes, cuya satisfacción se configura como la prioridad estratégica para garantizar la supervivencia empresarial. La calidad se contempla como el objetivo prioritario tanto de las actividades empresariales como de las profesionales.

d) En cuarto lugar debe señalarse como factor que esta influyendo en el sector de consultoría económica la dinámica de su propia evolución. Es decir, el crecimiento observado en la demanda de servicios que evidencian los datos de este sector, tanto en Europa como en España. De los datos facilitados desde la Asociación Española de Empresas de Consultoría (AEC, 2004 y 2005), es destacable el crecimiento generalizado de la consultoría económica debido a un resurgimiento de la tendencia a la externalización de determinadas actividades de las empresas. El último informe anual publicado por la Federación de Empresas Europeas de Consultoría (FEACO) denota un comportamiento más exigente de 
los clientes y una mayor orientación a los resultados. Los consultores que busquen mayor cuota de mercado deben proveerse de un servicio más completo.

\section{METOdOLOGÍA DEL ESTUDIO EMPÍRICO}

Tras realizar una revisión teórica de los factores que caracterizan el entorno de la consultoría y analizar la evolución de este mercado a través de los datos estadísticos disponibles, hemos llevado a cabo un estudio empírico en nuestra región: Cantabria (España) para conocer directamente la opinión de las partes implicadas (empresas y consultorías) en relación con los nuevos retos a los que habrán de enfrentarse, así como los cambios, mejoras e innovación que se deben implantar en la organización, en los propios profesionales y en los servicios ofrecidos.

Para ello, se ha realizado un doble estudio empírico basado en dos encuestas de opinión. Una analiza las necesidades que tienen las empresas de contar con los despachos de consultoría económica así como su grado de satisfacción con los mismos. La otra estudia como se satisfacen aquellas necesidades por parte de las consultoras así como su percepción por los cambios, mejoras e innovaciones que se deben introducir a la luz de las nuevas demandas.

\subsection{Objetivos} siguientes:

Los principales objetivos del estudio empírico realizado pueden resumirse en los

a) Detectar los distintos niveles de información contable financiera, fiscal y de gestión requeridos a las consultoras, según el tamaño, estructura y actividad realizada por la empresa.

b) Diferenciar aquellas necesidades contables que se satisfacen por el personal de la propia empresa, de aquellas otras necesidades que se satisfacen a través de personal externo a la empresa, es decir a través de despachos profesionales de consultoría, asesoría y otros de naturaleza económica análoga. Y detectar las expectativas insatisfechas.

c) Captar la opinión que tienen las empresas sobre los profesionales y la calidad de los servicios recibidos.

d) Conocer como se satisfacen los servicios requeridos por las empresas desde el punto de vista de los despachos profesionales así como las innovaciones y mejoras que estos consideran necesario introducir ante los cambios que se están produciendo en este mercado.

\subsection{Los cuestionarios y el muestreo estadístico}

La metodología utilizada este estudio, como se ha indicado anteriormente, se basa en la confección y realización de dos cuestionarios uno dirigido a las empresas y el otro a los profesionales de la consultoría, que guardan un paralelismo en las preguntas realizadas.

Se trata de un estudio empírico de tipo descriptivo y comparativo realizado en el período comprendido entre junio de 2004 a enero de 2005. El cuestionario (compuesto de 46 preguntas para las consultorías y 40 para las empresas), se estructuró en cuatro bloques de preguntas que corresponden a los siguientes: Las de tipo formal y organizativo (forma 
jurídica, composición, dotación de medios, nivel de ocupación, funcionamiento), las vinculados a los profesionales (nivel de formación, grado de responsabilidad e independencia, ética), las referidas a los servicios prestados (catálogo, grado de satisfacción de los clientes) y, por último, las que se corresponden con los clientes y el mercado (tipo de clientela, caracterización del mercado).

Dada la amplia población objeto de estudio 35.649 empresas (INSTITUTO NACIONAL DE ESTADÍSTICA, 2004) y 442 profesionales de la consultoría económica a finales de citado año, el análisis se efectuó mediante la selección de una muestra aleatoria estratificada de afijación proporcional para cada colectivo, fijando un nivel de confianza estadístico del 95\%. Los datos obtenidos fueron procesados utilizando los paquetes estadísticos SPSS y el programa integral DYANE. La presente investigación aplicada puede clasificarse en aquellas de tipo descriptivo transversal, incluyendo análisis cuantitativo y cualitativo.

\section{RESULTADOS DE LA ENCUESTA REALIZADA A LAS EMPRESAS SOBRE LAS NECESIDADES DE SERVICIOS DE CONSULTORÍA}

Seguidamente pasamos a mostrar los principales resultados obtenidos en el estudio empírico realizado a las empresas.

\subsection{Empresas encuestadas}

El tamaño de la empresa se ha medido principalmente en función del número de trabajadores y del volumen de facturación anual. Las Gráficas 1, 2 y 3 muestran la distribución y características de las empresas encuestadas.

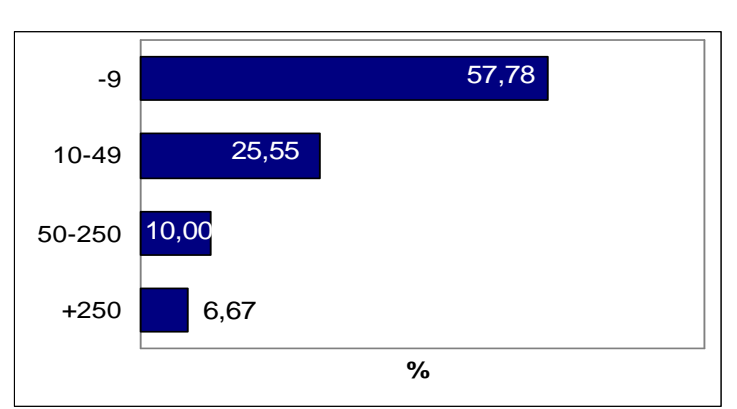

Gráfica 1 - Empresas según número de trabajadores

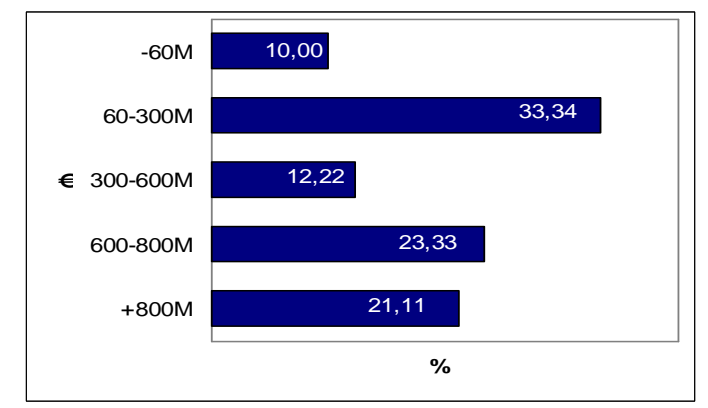

Gráfica 2 - Empresas según la facturación anual

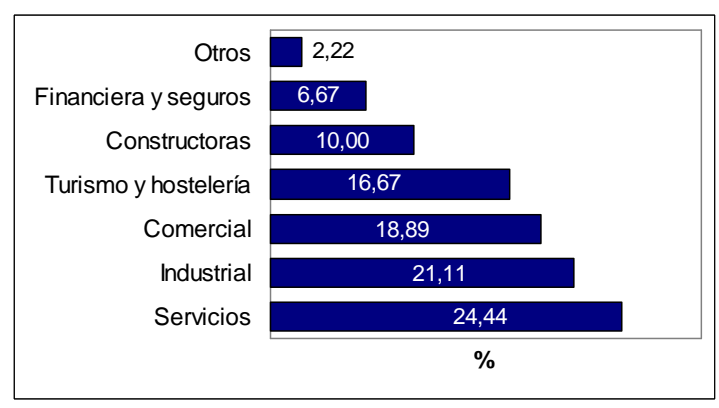

Gráfica 3 - Sectores encuestados 


\subsection{Necesidades de externalización de funciones administrativas y de gestión de las empresas}

En las empresas se evidencia una fuerte dependencia de los despachos profesionales para cubrir sus necesidades administrativas y de gestión. Se observa una relación directa entre el tamaño de la empresa y la necesidad de externalizar determinadas funciones a los profesionales de las consultorías (Gráfica 4 y Gráfica 5).

Como puede observarse, a menor dimensión de la entidad, mayor es la dependencia y las necesidades constantes de los servicios de la asesoría externa, ya sea para la mecanización o la llevaduría de la contabilidad y fiscalidad, elaboración de las nóminas y seguros sociales, para el cumplimiento de los documentos necesarios para satisfacer las obligaciones empresariales u otras funciones de gestión.

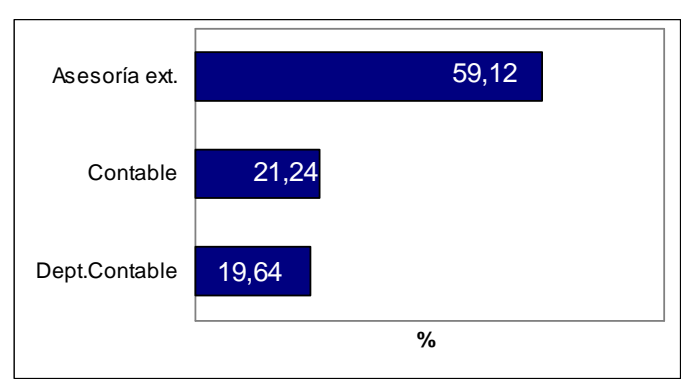

Gráfica 4 - ¿Quién se encarga de la contabilidad?

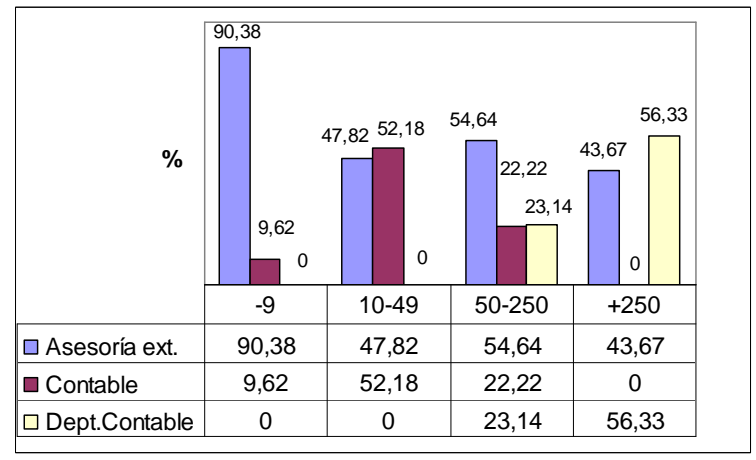

Gráfica 5 - Encargado de contabilidad según el $n^{\circ}$ de trabajadores

\subsection{Servicios demandados a las consultorías}

a) Necesidades en las micro y pequeñas empresas

- Se ha evidenciado que las empresas pequeñas con menos de 9 trabajadores y menos de 300.000 euros de facturación, demandan mayoritariamente los servicios de asesoramiento contable y fiscal, elaboración de la contabilidad, y confección de las nóminas y seguros sociales.

- Los servicios relacionados con la contabilidad interna y de gestión, no son habitualmente requeridos por las pequeñas entidades. Sin embargo, se observa que sí existe demanda en estas empresas en relación con la implantación de sistemas informatizados para la facturación, el control de existencias y la obtención de registros contables y fiscales que, además, permitan obtener el soporte documental exigido por el Fisco.

- Las principales críticas sobre los servicios recibidos por las asesorías son: la falta de información sobre posibles subvenciones, incentivos y otras ventajas a las que puedan acogerse. Manifiestan que las asesorías se limitan al cumplimiento de las obligaciones contables, fiscales, y laborales a las que están sujetas, sin alertarles de posibles ventajas o inconvenientes sobre cuestiones de interés para mejorar la rentabilidad o la gestión de su negocio.

b) Necesidades en las medianas y grandes empresas 
- Las empresas con más de 10 trabajadores y más 600.000 euros de facturación tienen su propio contable o profesional y cuando su tamaño aumenta, disponen de un departamento de contabilidad y/o fiscalidad que les permite elaborar y mecanizar la contabilidad, así como cumplimentar los documentos obligatorios a realizar que deben presentar conforme al calendario fiscal.

- Los servicios más solicitados son: el puro asesoramiento contable fiscal, laboral y mercantil. También estudios de mercado, valoraciones, análisis económico, financiero y de rentabilidad y, en general, la contabilidad para la gestión, sin perjuicio de los servicios clásicos de liquidación o supervisión del impuesto de sociedades y de las cuentas anuales.

- Además se observa un interés que sobrepasa la consultoría tradicional requiriendo servicios relacionados con los sistemas de información para la gestión y nuevas tecnologías que se concretan en solicitar paquetes integrados que permitan satisfacer a tiempo real el cálculo de costos, tiempos ociosos y localización de los bienes entre otros aspectos.

\subsection{Número de despachos de los que se reciben servicios profesionales}

Lo más frecuente es depender de uno solamente, existiendo una relación contractual fija. Pero hay empresas que dependen de dos despachos o incluso de tres. Estos despachos se refieren a los que ofrecen asesoramiento contable y fiscal por un lado, nóminas y seguros sociales por otro, y servicios de informática o asesoramiento jurídico en los casos que proceda, sin que con estos últimos se acuerden con carácter general contratos fijos. Solo el $2,41 \%$ de la muestra, no tenía contratos fijos con ningún despacho. La Gráfica 6 muestra mayor detalle a este respecto.

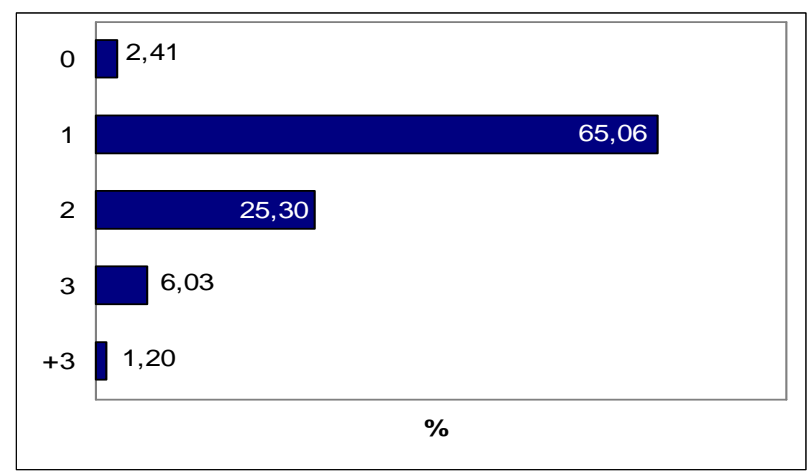

Gráfica 6 - Número de despachos de los que se reciben servicios

La mayor parte de las empresas han manifestado el deseo de recibir servicios integrales, en el sentido de que un solo despacho pueda satisfacer sus necesidades globales de asesoramiento. No obstante, hay empresas que por razones del sector al que pertenecen, disponen de agrupaciones empresariales que ofrecen determinados servicios tales como: asesoramiento contable, elaboración de nóminas y seguros sociales o, un servicio de asesoría jurídica. Estos servicios colectivos están dando lugar a que las empresas disfruten de los mismos y, solamente se vean en la necesidad de contratar aquellos otros servicios que no les ofrece la organización del sector. 


\subsection{Calidad de los servicios}

En nuestros días, existe un amplio consenso en las investigaciones realizadas (PARASURAMAN, ZEITHAML y BERRY, 1985; GRÖNROOS, 1984; ZEITHAML, 1988; GIL, 1994) en conceptuar la calidad de servicio como un atributo subjetivo de orientación externa; es decir, basada en las percepciones del cliente. De ahí que, para determinar o evaluar la calidad de servicio es imprescindible contar con las percepciones que tienen los clientes del servicio recibido. Es decir, es necesario obtener las valoraciones que hace el cliente de la excelencia del servicio, incluyendo dichas valoraciones no sólo los resultados obtenidos sino también el proceso de prestación del servicio. Y, es precisamente de la comparación entre expectativas del cliente y resultado actual recibido del servicio, de donde surgen o emanan las percepciones del cliente. Por ello, en este estudio la calidad de los servicios prestados por los despachos profesionales a las empresas se ha evaluado mediante el análisis de cuatro dimensiones: Grado de satisfacción con los servicios obtenidos; Grado de satisfacción con el asesor; Relación calidad-precio y, Expectativas insatisfechas.

\subsubsection{Satisfacción con los servicios obtenidos}

Como muestra la Gráfica 7, el 90,24\% de la totalidad de los encuestados manifiesta satisfacción con los servicios obtenidos por su asesor (valoración buena o muy buena) y, por tanto, sólo un $9,76 \%$ de los mismos se muestra descontento con los servicios que le prestan (valoración mala o regular).

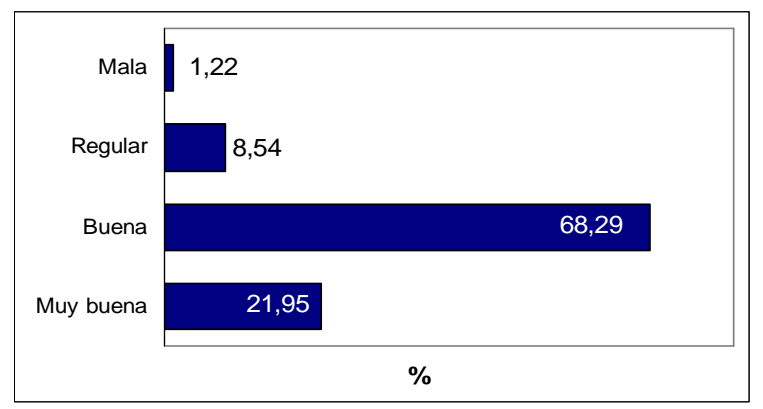

Gráfica 7 - Opinión sobre calidad de los servicios

\subsubsection{Satisfacción con el asesor}

La calidad de los servicios obtenidos se ratifica que es buena, al comprobar que no existe una excesiva rotación de asesores, si no que las empresas se muestran fieles a sus asesores con el paso del tiempo (Gráfica 8). Además, esta fidelidad no se ve motivada por la obligación de continuar con el mismo asesor por conocer los datos de la empresa, por los años de relación o por el mero miedo a cambiar de asesor, sino que la continuidad está basada en la voluntad de continuar con el mismo asesor por considerar que satisface las necesidades planteadas.

Asimismo, un porcentaje muy elevado de encuestados manifiesta que su asesor le atendió puntualmente ante los problemas en los que requirió sus servicios profesionales (Gráfica 9). 


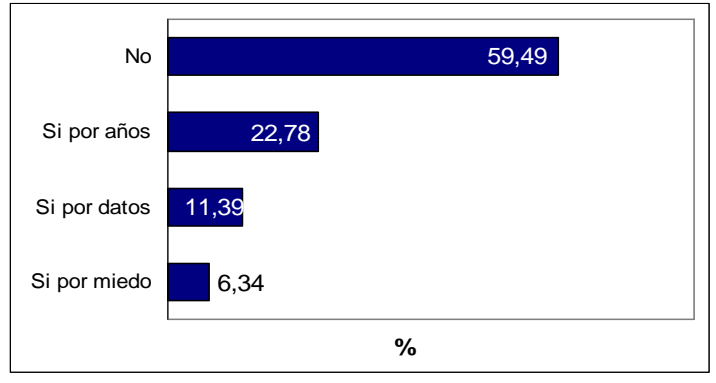

Gráfica 8 - ¿Se siente forzado a seguir con su asesor?

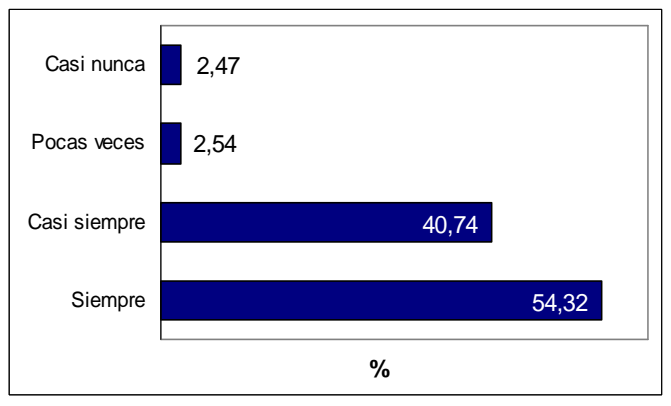

Gráfica 9 - Atención del asesor cuando surgen problemas

\subsubsection{Relación calidad-precio}

De las respuestas recibidas se desprende que atendiendo únicamente al precio de los servicios recibidos, un grupo considerable de encuestados los considera elevados. No obstante, cuando se preguntó por la opinión sobre los honorarios teniendo en cuenta la calidad de los servicios recibidos la respuesta cambió de tendencia, obteniéndose un 89,02\% de encuestados que calificaron de buenos o muy buenos los servicios obtenidos frente a sólo un 10,98\% que lo calificó como malo o regular (Gráfica 10 e Gráfica 11).

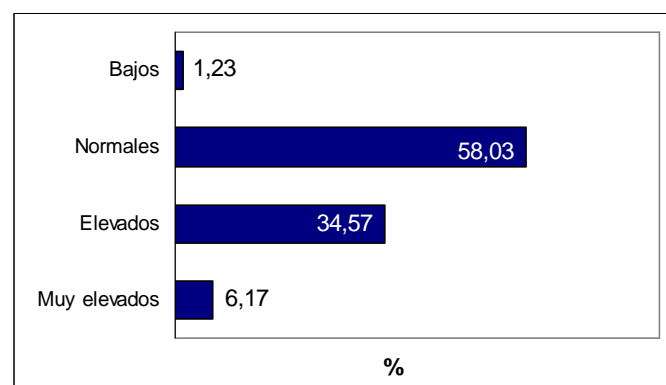

Gráfica 10 - Opinión sobre honorarios

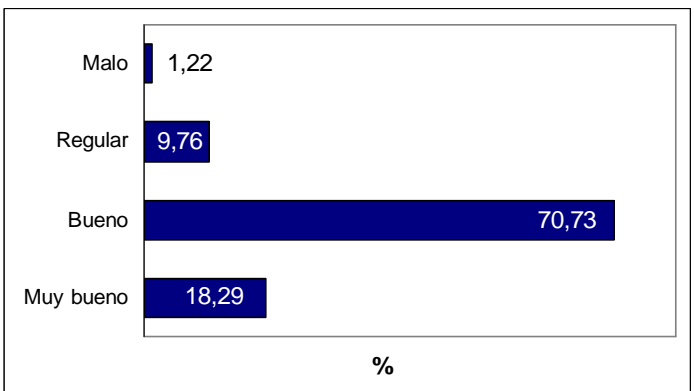

Gráfica 11 - Precio-calidad de los servicios

En consecuencia, del estudio empírico realizado sobre este aspecto, puede decirse que las empresas se muestran contentas en la relación calidad/precio con los servicios suministrados por los profesionales aunque algunas consideren algo elevados sus honorarios.

\subsubsection{Expectativas insatisfechas}

Derivado de las principales observaciones de carácter negativo, realizadas por algunos grupos de encuestados, se destaca lo siguiente:

a) La necesidad de alertar y prevenir a los empresarios ante los nuevos cambios o expectativas de modo que puedan planificar o adaptar sus negocios a los nuevos tiempos. Se evidencia también, la necesidad de dar mayor información a las empresas. Es decir las empresas estarían más satisfechas si los asesores además de suministrarles los servicios tradicionales, les informasen de nuevos planteamientos empresariales, de posibles alternativas para disfrutar de facilidades financieras, de incentivos fiscales o laborales a las que pudiesen acogerse, bien sean para disminuir sus costes, aumentar su rentabilidad o mejorar su administración o gestión. 
b) Se detecta, en muchos casos, la falta de preparación de los profesionales de la consultoría económica para dar servicios en el campo de la informática de gestión, nuevas tecnologías y sistemas, debiendo las empresas que requieran estos servicios acudir a empresas especializadas en el campo de la informática, que muchas veces carecen de una visión económica que permita sincronizar los beneficios derivados de un adecuado asesoramiento de ambos campos profesionales.

\section{RESULTADOS DE LA ENCUESTA REALIZADA A LAS CONSULTORÍAS}

Seguidamente pasamos a mostrar los principales resultados obtenidos en la encuesta realizada a los despachos de consultoría económica.

\subsection{Distribución de la muestra, perfil de los profesionales y forma jurídica de los despachos de consultoría}

La muestra se ha seleccionado teniendo en cuenta todos los despachos de consultoría con domicilio en la región de Cantabria. La media de los profesionales encuestados corresponde a profesionales que llevan ejerciendo más de quince años. Esto es debido a que los cuestionarios se dirigieron, según la planificación del estudio, a los socios titulares de los despachos profesionales, y éstos en su mayoría, llevan varios años en funcionamiento.

La mayoría de los encuestados tienen una edad que oscila entre los 35 y los 50 años seguidos de aquellos que tienen entre 50 y 65 años. No obstante, también se observa que hay despachos de reciente constitución, con menos de cinco años, formados por profesionales jóvenes cuya edad se sitúa entre 20 y 35 años.

\subsection{Cambios, mejoras e innovaciones realizadas en los despachos de consultoría}

Los principales cambios, mejoras e innovaciones que se han detectado en las respuestas recibidas del estudio empírico realizado, los hemos agrupado en los epígrafes que se muestran seguidamente.

\subsubsection{Estructura y composición de los despachos profesionales}

Se observa un importante incremento de despachos en la región estudiada, en los últimos tres años. Muchos de ellos corresponden a sucursales de la misma entidad establecidos en diferentes municipios. La forma jurídica habitualmente adoptada para la prestación de servicios son sociedades mercantiles, anteriormente predominaban los profesionales autónomos.

En relación con la estructura de los despachos no pueden hacerse generalizaciones debido a la gran variedad y diferencias en su composición. Sin embargo se ha observado un cambio de despachos unipersonales a despachos con gran número de trabajadores. Asimismo la formación de estos trabajadores es muy diferente en cada asesoría o despacho profesional. Se observan una tendencia hacia despachos con profesionales de naturaleza multidisciplinar en los que coexisten economistas, diplomados empresariales, abogados, graduados sociales, informáticos, etc. que permiten el asesoramientos en los diferentes campos que abarca la extensa profesión de la consultoría económica.

\subsubsection{Cualificación, formación continuada y equipamiento}


El estudio realizado, muestra que existe preocupación por actualizar conocimientos y estar al día de los cambios e innovaciones. Así lo demuestra la gran mayoría de encuestados en relación con los numerosos cursos realizados por año así como por las revistas especializadas a las que están suscritos. Todo ello sin perjuicio del tiempo dedicado al estudio de temas generales y específicos para atender asuntos concretos que requieren los clientes.

El interés por la actualización también se observa en el equipamiento y la utilización de las tecnologías de la información y las comunicaciones. La mayoría de los encuestados ha realizado cursos de naturaleza informática, está plenamente generalizado el uso del ordenador e Internet como herramienta básica para sus funciones habituales. La mayoría tiene un ordenador por persona y los equipos informáticos se renuevan mayoritariamente entre dos y cuatro años. La mayoría manifiestan tener sistemas de seguridad y protección de datos.

\subsubsection{Servicios de consultoría}

Los servicios están evolucionando por un lado en rapidez gracias a las nuevas tecnologías y por otro lado en el ofrecimiento de nuevos servicios relacionados con operaciones financieras y servicios de gestión. No obstante la principal líneas de mercado continúa siendo la consultoría tradicional, véase tabla siguiente.

Tabla 1 - Servicios prestados según el volumen de facturación

\begin{tabular}{||l|c|}
\hline \multicolumn{1}{|c|}{ SERVICIOS PRESTADOS } & \% FACTURACIÓN \\
\hline \hline Asesoramiento contable y fiscal & 42,31 \\
\hline Elaboración de contabilidad & 19,65 \\
\hline Laboral & 15,00 \\
\hline Auditoría de cuentas & 8,80 \\
\hline \hline Otros trabajos de consultoría & 5,74 \\
\hline Intervenciones judiciales. & 5,35 \\
\hline Sistemas de información de gestión & 2,10 \\
\hline Contabilidad de costes & 1,05 \\
\hline \hline
\end{tabular}

\subsection{4 Ética, competitividad y responsabilidad}

En la época actual a los profesionales les afecta de forma muy significativa la responsabilidad que deben asumir al contratar trabajos profesionales, de tal modo que la falta de conocimientos podría influirles de forma decisiva para rechazar determinados trabajos incluso cuando aquellos estén bien retribuidos y el nivel de ocupación del profesional no alcance el cien por cien de su capacidad laboral (Gráficas 12 y 13). A estos efectos, debe señalarse el incremento en las demandas interpuestas exigiendo la depuración de responsabilidades de los profesionales, las importantes sanciones previstas en la legislación vigente, así como el Código de Ética de la Asociación Española de Consultores (AEC) que establece como falta para sus asociados el aceptar un trabajo para el que no estén capacitados. 


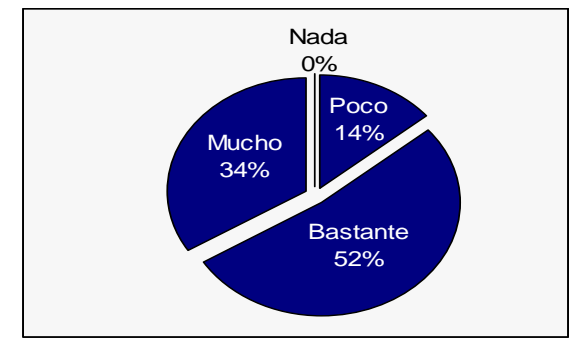

Gráfica 12 - Influencia de la responsabilidad

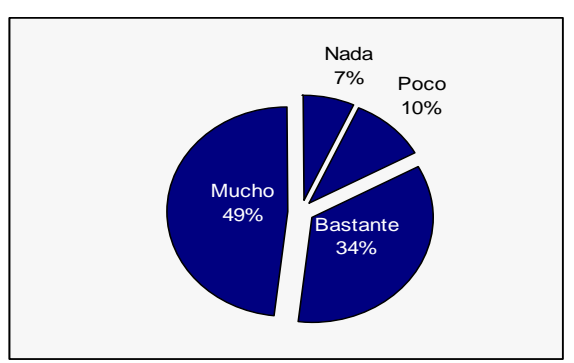

Gráfica 13 - Influencia de la falta de conocimientos

Como aspectos negativos del sector destacan el intrusismo laboral (el 93\% lo califican de mucho y bastante), especificándose por algunos que sería conveniente definir con mayor precisión, las funciones o actividades que corresponde a cada nivel de titulación que puede ofrecer servicios de consultoría económica.

\section{CONCLUSIONES}

Al margen de las limitaciones propias de los trabajos empíricos (errores de muestreos y otros relacionados con la personalidad, formación o edad de los encuestados. El hecho de que las diferencias encontradas en los valores medios obtenidos sean en algunas cuestiones muy pequeñas, supone la existencia de unos criterios comunes que permiten extraer las siguientes conclusiones:

a) A través del estudio de las necesidades de las empresas se detecta lo siguiente:

- La existencia de una fuerte dependencia tanto en las funciones de administración como en las de gestión y dirección de las empresas en relación con los despachos de consultoría económica. Si bien, las necesidades de asesoramiento económico difieren según el tamaño de las empresas.

- Se solicitan servicios de asesoramiento integrales de modo que un solo despacho pueda satisfacer la totalidad de los servicios demandados, aunque en ocasiones se requieran servicios muy especializados.

- El grado de satisfacción con las consultorías es adecuado si bien, existen nuevas demandas no cubiertas relacionadas con la tecnología de la información y, en general con los servicios de consultoría de gestión. Es necesario seguir mejorando en la calidad de los servicios que permita no solo cumplir con las obligaciones empresariales sino también anticiparse en el tiempo para que las empresas puedan prever y acomodar su gestión a los nuevos retos que va planteando el mundo de los negocios.

b) Para hacer frente a estas necesidades los nuevos retos o desafíos que deben realizar los despachos profesionales de la consultoría económica son los siguientes:

- Ampliar del numero de oficinas de una misma compañía (en la provincia y en otras ciudades españolas e incluso fuera de España o buscar colaboraciones entre consultoras) para acercar los servicios ofrecidos a las empresas. 
- Continuar en la mejora del equipamiento y modernización de las instalaciones y de los medios técnicos propios de la consultoría.

- Creación de despachos multidisciplinares que permitan ofrecer servicios integrales o adquirir una gran especialización en aquellas áreas que puedan constituir nuevos nichos de mercado.

- No cesar en la preocupación por la necesidad de adquirir formación permanente, y adiestramiento en nuevas tecnologías de la información que permita asesorar de forma más rápida y eficiente.

- Tener en cuenta además de los servicios clásicos de consultoría, el aumento de la demanda hacia las funciones de gestión sobre las meramente administrativas o formales.

- Fomentar el comportamiento ético, la mayor escrupulosidad en la elaboración de tareas e insistir en adoptar las medidas para salvaguardar la responsabilidad profesional asumida en los trabajos.

- Adopción de medidas para combatir el intrusismo laboral además de insistir en llegar a una definición y acotamiento legal de las funciones del asesor económico según los diferentes niveles de titulación que se posea.

Sin duda, los cambios comentados y los nuevos desafíos que están afectando a este sector son palpables desde hace ya algunos años y su asunción por las empresas de consultoría económica resulta necesario, ello explica las mejoras introducidas cuando menos, para el mantenimiento de su posición competitiva.

\section{REFERENCIAS}

ALONSO, E.; GIL, I. Cómo relacionarse en el trabajo: teoría y práctica de la consultoría. . Madrid: Prentice Hall - Financial Times, 2005.

AMADO GUIRADO, J.; HUETE, L. Mª . Asesorías del siglo XXI. SA: CISS PRAIS, 1999.

AMAT, Oriol; BLAKE, John. Contabilidad Europea. Madrid: Monografías (AECA), 1996.

APPELBAUM, S. H.; STEED, A. J. The critical success factors in the client-consulting relationship. The Journal of Management Development. v. 24, $\mathrm{n}^{\circ}$ 1/2, p. 68-93, 2005. http://dx.doi.org/10.1108/02621710510572362

ASOCIACIÓN ESPAÑOLA DE ASESORES FISCALES. El asesor fiscal ante el nuevo siglo. Madrid, 2000.

ASOCIACIÓN ESPAÑOLA DE EMPRESAS DE CONSULTORÍA (AEC): La Consultoría en España. Madrid: AEC, 2003, 2004 y 2005. Puede verse en: <http://www.consultoras.org/frontend/plantillaaec/base.php>.

CARRO, M.; HERNANDO, G. El sector de consultoría económica y auditoría de cuentas en Cantabria. Situación actual y tendencias futuras. Colegio de Economistas y Colegio de Titulados Mercantiles y Empresariales de Cantabria, 1995.

DAWES, P.P.; DOWLING, G.; GRAHAME, R. ; PATTERSON, P.P. Criteria used to select management consultants. Industrial Marketing Management, v. 21, n. 3, p.187-193, 1992. http://dx.doi.org/10.1016/0019-8501(92)90015-L 
DEMBITZ, A.; ESSINGER, J. Breakthrough consulting: so you want to be a consultant? Turn your expertise into a successful consulting business. London/New Cork: Financial Times - Prentice Hall. 2000.

DIRECCIÓN GENERAL DE POLÍTICA DE LA PYME. Estadísticas Pyme Cantabria, 2004.

EASTON, T. A.; CONANT, R. W. Using consultants: a consumer's guide for managers. Chicago/Illinois: Probus Publishing, 1985.

FINCHAM, R. The agent's agent: Power, knowledge, and uncertainty in management consultancy. International Studies of Management \& Organization. v. 32, n. 4, p. 67-103, 2003.

GAY, D. L.; LABONTE, T. J. Consultoría del rendimiento: comenzamos. Training \& Development Digest, n. 45, p. 28-32, 2004.

GIL SAURA, I. La conceptualización y evaluación de la calidad de servicio al cliente percibida en el punto de venta. Club Gestión de Calidad, 1995.

GITLOW, H. S. A comparison of Japanese total quality control and deming's theory of management. The American Statistician. v. 48, n. 3. August, 1994. http://dx.doi.org/10.2307/2684716

GRÖNROOS, C. A service quality model and its marketing implications. European Journal of Marketing, v. 18, n. 4, 1984.

HISPALINK - CANTABRIA. La economía cántabra. 2004. Puede verse en: $<$ http://www.hispalink.org/cantabrias.htm>.

INSTITUTO DE CONTABILIDAD Y AUDITORÍA DE CUENTAS. Informe sobre la situación actual de la contabilidad en España y líneas básicas para abordar su reforma, 2002.

INSTITUTO DE CONTABILIDAD Y AUDITORÍA DE CUENTAS (ICAC). BOICAC $N^{o}$ 27: Comisión de la Unión Europea. Función, posición y responsabilidad civil del auditor legal en UE (96/C 321/01), 1996.

MAÑAS, E. La demanda de servicios a empresas según tamaño, actividad y localización. Papeles de Economía Española, no 50, $1^{\circ}$ trimestre, p. 307-311, 1992.

MCKENNA, Christopher D. The origins of modern management consulting. Business and Economic History, v. 24, n. 1, p. 51-58, 1995.

MERLI, G. Total Manufacturing management: la estrategia industrial en los años 90. . Madrid: Tecnologías de Gerencia y Producción, 1991.

MOLES y COGOLLUDO. Satisfacción del cliente y calidad de servicios. Monografías Asesorías: Gestión empresarial. Valencia: Editorial CISS, 1996.

PARASURAMAN, A.; BERRY, L. L.; ZEITHAML, V. A. A conceptual model of service quality and its implications for future research. Journal of Retailing, v.49, p.41-50, fall, 1985.

PARKINSON, C.N. In-Laws and Outlaws. Westport/CT: Greenwood Press, 1962. pp.166167. 
RAFAEL; José. Las empresas españolas de consultoría ante 2005. Puede verse en: $<\mathrm{http} / / / \mathrm{www}$.consultoras.org/frontend/plantillaAEC/seccion.php?id_seccion=107>.

RIBEIRO SORIANO, D. The impact of consulting service on Spanish firms. Journal of Small Business Management. Milwaukee, v. 41, n. 4, p. 409, Octubre, 2003.

RUIZ, A.; MEMBRADO, J. Los consultores del siglo XXI: evolución de los conceptos en la consultoría de la calidad. Qualitas hodie: excelencia, desarrollo sostenible e innovación, $\mathrm{n}^{\circ}$ 101, p. 15-17, 2004.

SCHUETZE, W.P. What are assets and liabilities? Where is true north? (Accounting that my sister would understand). Abacus, v. 37, n.1, p. 1-25, 2001. http://dx.doi.org/10.1111/1467$\underline{6281.00072}$

SETÓ PAMIES; D. La relación entre la calidad de servicio percibida por el cliente y la fidelidad de servicio. Revista. Europea de Dirección y Economía de Empresa. v.1 4, n. 1, p.141-156, 2005.

SHAPIRO, E. C.; ECCLES, R. G.; SOSKE, T. L. Consultoría: ¿es la solución parte del problema? Harvard-Deusto Business Review, n. 58, p. 38-45, 1993.

VARDUERTELES, I. Gestionando Pymes con sentido común. Madrid: CIE. Inversiones. Ed. Dossat, 2002.

WERR, A.; STYHRE, A. Management consultants-friend or foe? Understanding the ambiguous client-consultant relationship. International Studies of Management \& Organization. v.. 32, n. 4, p. 43-66, 2003.

WILLIAMS, R. Management fashions and fads: understanding the role of consultants and managers in the evolution of ideas. Management Decision, v. 42, n. 5/6, p.769-784, 2004. http://dx.doi.org/10.1108/00251740410542339 